\title{
Mapping of Micro-Tom BAC-End Sequences to the Reference Tomato Genome Reveals Possible Genome Rearrangements and Polymorphisms
}

\author{
Erika Asamizu, ${ }^{1}$ Kenta Shirasawa, ${ }^{2}$ Hideki Hirakawa, ${ }^{2}$ Shusei Sato, ${ }^{2}$ Satoshi Tabata, ${ }^{2}$ \\ Kentaro Yano, ${ }^{3}$ Tohru Ariizumi, ${ }^{1}$ Daisuke Shibata, ${ }^{2}$ and Hiroshi Ezura ${ }^{1}$ \\ ${ }^{1}$ Faculty of Life and Environmental Sciences, University of Tsukuba, 1-1-1 Tennodai, Tsukuba 305-8572, Japan \\ ${ }^{2}$ Kazusa DNA Research Institute, 2-6-7 Kazusa-kamatari, Kisarazu 292-0818, Japan \\ ${ }^{3}$ School of Agriculture, Meiji University, 1-1-1 Higashi-mita, Tama-ku, Kawasaki 214-8571, Japan \\ Correspondence should be addressed to Erika Asamizu, asamizu@gene.tsukuba.ac.jp
}

Received 10 August 2012; Accepted 18 October 2012

Academic Editor: Pierre Sourdille

Copyright ( 2012 Erika Asamizu et al. This is an open access article distributed under the Creative Commons Attribution License, which permits unrestricted use, distribution, and reproduction in any medium, provided the original work is properly cited.

\begin{abstract}
A total of 93,682 BAC-end sequences (BESs) were generated from a dwarf model tomato, cv. Micro-Tom. After removing repetitive sequences, the BESs were similarity searched against the reference tomato genome of a standard cultivar, "Heinz 1706." By referring to the "Heinz 1706" physical map and by eliminating redundant or nonsignificant hits, 28,804 "unique pair ends" and 8,263 "unique ends" were selected to construct hypothetical BAC contigs. The total physical length of the BAC contigs was 495,833 , $423 \mathrm{bp}$, covering $65.3 \%$ of the entire genome. The average coverage of euchromatin and heterochromatin was $58.9 \%$ and $67.3 \%$, respectively. From this analysis, two possible genome rearrangements were identified: one in chromosome 2 (inversion) and the other in chromosome 3 (inversion and translocation). Polymorphisms (SNPs and Indels) between the two cultivars were identified from the BLAST alignments. As a result, 171,792 polymorphisms were mapped on 12 chromosomes. Among these, 30,930 polymorphisms were found in euchromatin ( 1 per 3,565 bp) and 140,862 were found in heterochromatin ( 1 per $2,737 \mathrm{bp}$ ). The average polymorphism density in the genome was 1 polymorphism per 2,886 bp. To facilitate the use of these data in Micro-Tom research, the BAC contig and polymorphism information are available in the TOMATOMICS database.
\end{abstract}

\section{Introduction}

Tomato (Solanum lycopersicum) is one of the most important vegetable crops cultivated worldwide. Tomato has a diploid $(2 \mathrm{n}=2 \mathrm{x}=24)$ and relatively compact genome of approximately $950 \mathrm{Mb}[1]$. Recently, its genome has been completely sequenced by the international genome sequencing consortium [2].

Genetic linkage maps of tomato have been created by crossing cultivated tomato (S. lycopersicum) with several wild relatives, S. pennellii, S. pimpinellifolium, S. cheesmaniae, S. neorickii, S. chmielewskii, S. habrochaites, and S. peruvianum [3]. Introgression lines generated from a cross between $S$. lycopersicum and S. pennellii have contributed to the isolation of important loci and quantitative trait loci (QTLs) related to fruit size by utilizing DNA markers on the TomatoEXPEN 2000 genetic map [4-9]. Such interspecies genetic mapping is effective because the divergent genomes provide many polymorphic DNA markers. In contrast, intraspecies mapping is less popular in tomato because of the low genetic diversity within cultivated tomatoes that has resulted from the domestication process and subsequent modern breeding [10]. Recently, we developed SNP, simple sequence repeat (SSR), and intronic polymorphic markers using publicly available EST information and BAC-end sequences (BESs) derived from "Heinz 1706," a standard line for tomato genomics [11, 12], and applied these markers to create linkage maps between Micro-Tom and either Ailsa Craig, a greenhouse tomato, or M82, a processing tomato, by mapping 1,137 markers [12]. 
Micro-Tom, a dwarf cultivar, is regarded as a model cultivar for functional genomics of tomato because of several characteristics, including small size $(20 \mathrm{~cm}$ plant height), short life cycle (3 months), existence of indoor cultivation protocols under normal fluorescent conditions, and highefficiency transformation methods that have been developed for this line [13-15]. The dwarf phenotype of Micro-Tom is the result of mutations in at least two major recessive loci. dwarf $(d)$ encodes a cytochrome $\mathrm{P} 450$ protein, which functions in the brassinosteroid biosynthesis pathway [16]. Another locus, miniature (mnt), is suggested to associate with gibberellin (GA) signaling without affecting GA metabolism, but the causal gene has not been identified to date [17]. In Japan, Micro-Tom genomics resources have been extensively accumulated, mainly in the framework of the National BioResource Project (NBRP) (http://tomato .nbrp.jp/indexEn.html). Large-scale ethyl methanesulfonate (EMS) and gamma-ray-mutagenized populations have been created, and visible phenotype data have been accumulated [18-20]. The availability of Micro-Tom genome sequence data will accelerate the mapping of mutant alleles.

BAC-end sequencing has been performed in the tomato standard line "Heinz 1706" genome project to order BAC clones along the chromosomes [21]. Currently, about 90,000 BESs are available at the Sol Genomics Network (SGN, http://solgenomics.net/). BAC-end sequencing has been conducted for other crop species. In the rice indica cultivar "Kasalath," 78,427 BESs were generated from 47,194 clones and mapped onto the "Nipponbare" reference genome. As a result, 12,170 paired BESs were mapped that covered $80 \%$ of the rice genome [22]. Recently, BAC-end sequencing has been performed in crop plants with higher genome complexity. BESs from a commercial sugarcane variety, an interspecific hybrid with complex ploidy, were generated to analyze microsynteny between sugarcane and sorghum [23]. In wheat, which has a complex hexaploid genome, the short arm of chromosome $3 \mathrm{~A}$ was flow sorted to make a BAC library, and chromosome arm-specific BESs were generated for DNA marker development [24]. In switchgrass, more than 50,000 SSRs were identified from 330,000 BESs, and this enabled detailed analysis on the evolution of this species [25]. A low level of genetic variation has been observed for cultivated peanuts. Polymorphic SSRs were accumulated from the BESs and successfully used in the construction of a genetic map [26]. BAC-end sequencing can be useful as a resource for performing comparative genomic studies through mapping of the sequences to a reference genome and by facilitating the development of polymorphic DNA markers.

In the present study, we generated 93,682 single-pass end sequences from a Micro-Tom BAC library. To compare the structures between the reference tomato "Heinz 1706" genome, mapping of unique ends was performed, and possible genome rearrangements and polymorphisms were identified.

\section{Materials and Methods}

2.1. Micro-Tom BAC Library Construction. Micro-Tom (TOMJPF00001) seeds were obtained from the NBRP
(MEXT, Japan) and sent to the Clemson University Genomics Institute (CUGI) for BAC library construction. The genomic DNA was partially digested, and fragments were cloned into the Hind III site of pIndigoBAC536. A total of 55,296 clones in Escherichia coli DH10B cells were arrayed in 144 384-well plates.

2.2. End Sequencing of Micro-Tom BAC Clones. To analyze BESs, the BAC DNAs were amplified using a TempliPhi largeconstruction kit (GE Healthcare, UK), and the end sequences were analyzed according to the Sanger method, using a cycle sequencing kit (Big Dye-terminator kit, Applied Biosystems, USA) with a type $3730 x \mathrm{x}$ DNA sequencer (Applied Biosystems). The resulting sequence reads were quality checked with PHRED [27, 28], allowing the identification and removal of low-quality $(\mathrm{QV}<20)$ sequences. The 93,682 reads clearing the quality criteria were submitted to DDBJ/ GenBank with accession numbers FT227487-FT321168.

2.3. Mapping to the Reference Genome and Analyses. BES reads were subjected to similarity search using the BLASTN program $[29,30]$. To isolate unique sequences from repetitive ones, 93,682 BESs were searched against the repeat database in ITAG2.3 (http://solgenomics.net/) using a cutoff $E$-value of less than $10^{-50}$. The remaining sequences were searched against the published version of the "Heinz 1706" genome (SL2.40), which was accessed from the SGN database (http://solgenomics.net/). From all of the BLAST alignments, BESs were extracted according to the following criteria, suggested in a previous report [22]: (1) sequence identity $>$ $90 \%$ and alignment coverage $>50 \%$; (2) mapped positions of each pair of ends $<200 \mathrm{~kb}$ apart in the same chromosome; (3) direction of each paired end is correct; (4) BLASTN $E<$ $10^{-100}$; (5) a minimum of one hit for one of the paired ends; (6) no redundant chromosomal locations. Sequence polymorphisms (SNPs and Indels) between Micro-Tom and "Heinz 1706" were predicted based on the BLASTN alignment. Since we did not allow a gap exceeding 27 bases, only Indels up to 26 bases in length were counted.

2.4. Database and Clone Distribution. Mapped data and SNP/Indel sites were made accessible through the database TOMATOMICS at http://bioinf.mind.meiji.ac.jp/tomatomics/. BAC clones are available upon request from NBRP tomato (http://tomato.nbrp.jp/indexEn.html).

\section{Results}

3.1. General Features of the Generated BESs. The BAC insert size distribution was deduced based on the mapping results. According to these results, $45.4 \%(6,396$ out of 14,101$)$ of the BACs ranged from 100 to $120 \mathrm{~kb}$, with average and median sizes of $101.3 \mathrm{~kb}$ and $101.8 \mathrm{~kb}$, respectively (Figure 1). By multiplying by the number of clones $(55,296)$, this BAC library covers $5.9 \mathrm{x}$ of the $950 \mathrm{Mb}$ tomato genome.

Micro-Tom BES mapping to the "Heinz 1706" genome was processed as indicated in Figure 2. By eliminating repetitive, redundant, and unmapped sequences, 28,804 "unique 


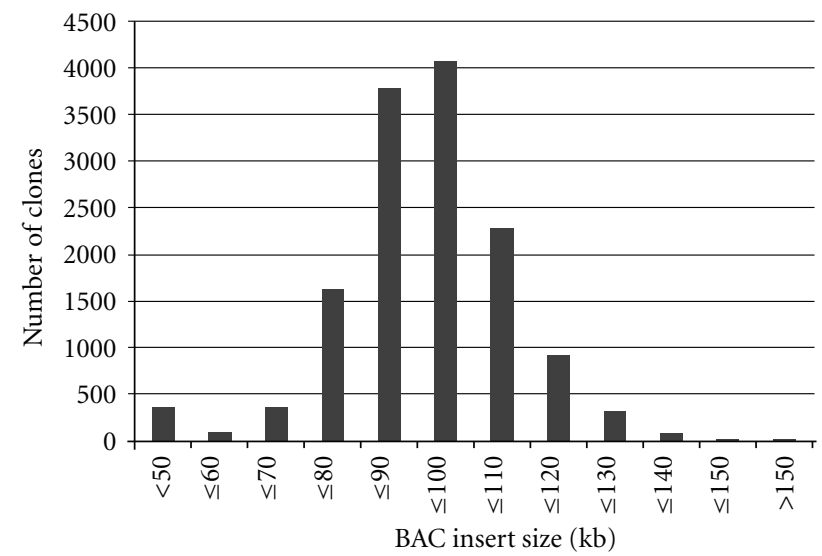

FIGURE 1: Distribution of BAC clone insert size. The insert size was deduced by mapping BESs onto the reference "Heinz 1706 " genome (SL2.40).

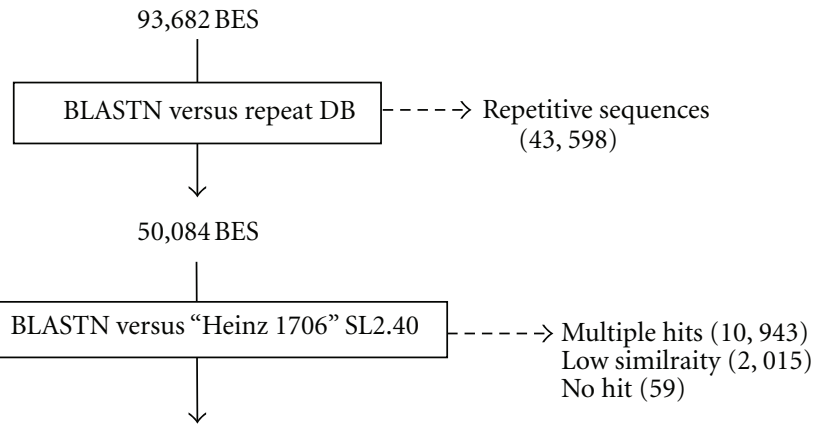

$37,067 \mathrm{BES}$

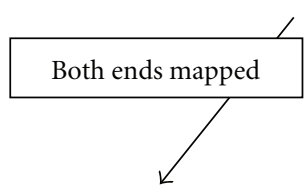

$28,804 \mathrm{BES}$

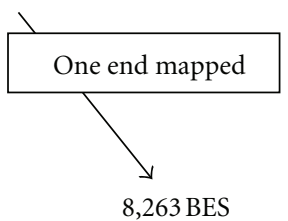

FIGURE 2: Flow of the BES analysis. To eliminate repetitive sequences, 93,682 BESs were initially searched against the repeat dataset of ITAG 2.3 with a BLASTN cutoff value of $E<10^{-50}$. Next, the remaining sequences were mapped onto the "Heinz 1706" pseudomolecule sequences (SL2.40) under the following criteria: identity $>90 \%$, coverage $>50 \% ; E<10^{-100}$; the inclusion of single hits only. Mapped BESs were classified as either unique pair ends, for which both ends were mapped, or unique ends, for which only one end was mapped.

pair ends" and 8,263 "unique ends" were selected. Paired-end sequences were mapped onto the reference tomato genome sequence, and 2,248 hypothetical BAC contigs were constructed (see details at TOMATOMICS, http://bioinf.mind .meiji.ac.jp/tomatomics/). The integrity of the hypothetical contigs was confirmed by linking to the DNA markers on two genetic maps, $\mathrm{AMF}_{2}$ and $\mathrm{MMF}_{2}$ (see Supplementary Table 1 in Supplementary Material available online at doi:10.1155/2012/437026).

The genome coverage of the hypothetical BAC contigs was assessed by applying euchromatin/heterochromatin boundary information from the genetic map EXPEN2000 [2]. The results indicated that the euchromatin coverage ranged between $45.1 \%$ and $71.1 \%$ (average, 58.9\%) among the different chromosomes, while heterochromatin coverage ranged between $57.4 \%$ and $75.3 \%$ (average, $67.3 \%$ ). The total physical length of the BAC contigs was $495,833,423 \mathrm{bp}$, covering $65.3 \%$ of the total chromosomes (Table 1).

3.2. Possible Genome Rearrangements. To assess the occurrence of genome rearrangements, Micro-Tom and the reference tomato "Heinz 1706" were compared. Possible inversions, translocations, and insertions were considered. To eliminate an artificial effect (e.g., chimeric BAC clones), only regions covered by more than two BAC clones were selected. After removing regions that had cleared the criteria for extraction (see Section 2 ) but were either shown to be multicopy by manual evaluation of the BLAST results or displayed similarity to transposable elements, we obtained two cases of a possible rearrangement between Micro-Tom and "Heinz 1706" (Table 2). On chromosome 2, a possible inversion was detected. The size of this inversion could be $20-220 \mathrm{~kb}$ 

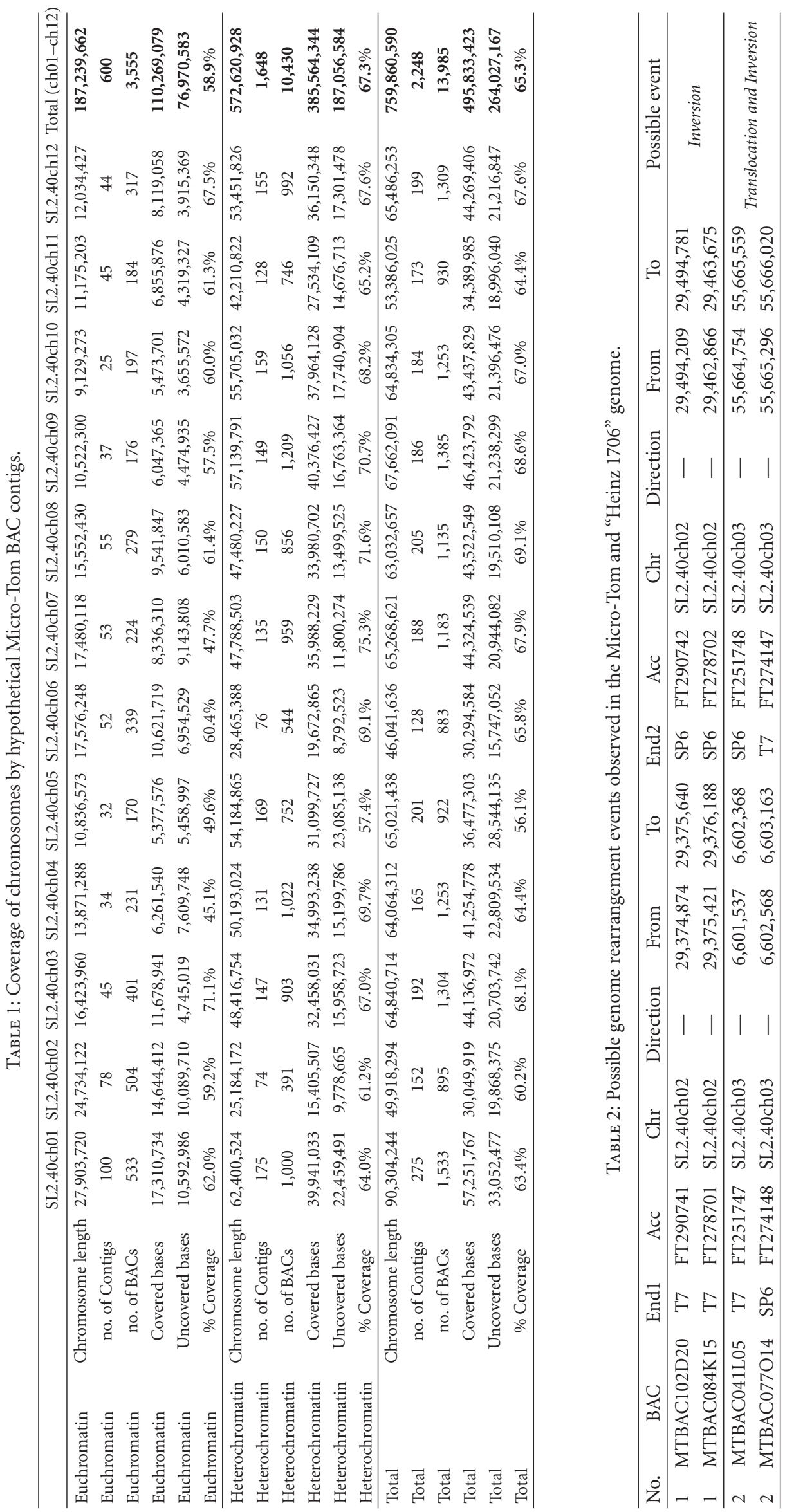
depending on which end of the BAC clone is inversed. Translocation and inversion were observed on chromosome 3. For each of two BAC clones (MTBAC041L05 and MTBAC077O14), one of the ends was mapped to $6,601 \mathrm{~kb}$ of chromosome 3, while the other end was mapped to $55,665 \mathrm{~kb}$, more than 49 megabases apart. In addition, both ends were mapped on the minus strand.

\subsection{Polymorphisms between Micro-Tom and the Reference} Tomato. SNPs and Indels between Micro-Tom and "Heinz 1706 " were identified. Among the SNPs and Indels found, 171,792 were mapped on 12 chromosomes, and 2,635 were mapped on pseudomolecules with no chromosomal information (SL2.40ch00 of the tomato whole-genome shotgun chromosomes) (Table 3 and Supplementary Table 2, see details at TOMATOMICS). According to these results, among the mapped SNPs and Indels, a total of 30,930 polymorphisms were found in the euchromatin (1 out of $3,565 \mathrm{bp}$ ), and 140,862 were found in the heterochromatin (1 out of $2,737 \mathrm{bp}$ ). The average polymorphism density in the genome was 1 polymorphism per $2,886 \mathrm{bp}$. Transversiontype SNPs were observed in 83,262 cases, while 60,631 were transition-type SNPs. Among the 30,534 Indels, single-base insertions (on the SL2.40 version of the tomato wholegenome shotgun chromosomes) were observed in 10,740 cases, and single-base deletions were seen in 17,064 cases. The remainder were larger Indels, ranging from 2 to $26 \mathrm{bp}$ (Supplementary Table 2). Classification of polymorphisms regarding genic or intergenic regions is shown in Table 4.

\section{Discussion}

By selecting unique end sequences from 93,682 reads, 28,804 paired ends (14,402 pairs) and 8,263 unpaired ends were obtained. The majority of the nonselected sequences $(43,598)$ were derived from repetitive regions. For the rest, 10,943 had redundant hits to the "Heinz 1706" genome, possibly including repetitive sequences that were not represented in the repeat database in ITAG2.3 (http://solgenomics.net/), 2,015 showed weak similarity, and 59 showed no similarity (Figure 2). Considering that the genome has been previously estimated to be composed of $25 \%$ gene-rich euchromatin $[31,32]$, BES selection in this study $(39.6 \%,(28,804+$ $8,263) / 93,682)$ ) could have eliminated repetitive regions to a moderate degree. We identified 59 reads showing no significant similarity to the "Heinz-1706" genome. Micro-Tom was bred by crossing the home-gardening cultivars, Florida Basket and Ohio 4013-3. The pedigree of Ohio 4013-3 suggested that a wild relative species was used in the breeding history $[18,33]$. Such introgressed segments may lead to the introduction of genomic regions not harbored by "Heinz 1706." The Micro-Tom genome is now being sequenced (draft sequence data available at DDBJ with the accession number DRA000311), and mapping of orphan BESs to the de novo assembly of Micro-Tom genome data will help to clarify this question.

The total physical length of Micro-Tom BAC contigs was $495,833,423 \mathrm{bp}$, which covers approximately $65.3 \%$ of the
DNA from all 12 chromosomes. In the Kasalath rice BES analysis, chromosomal coverage in relation to the reference Nipponbare pseudomolecule was about $80 \%$, despite the lower number $(78,427)$ of analyzed BESs [22]. Because we used the same criteria for repetitive sequence selection $(E<$ $10^{-50}$ ), the discrepancy between the two studies might be due to the larger genome size of tomato $(950 \mathrm{Mb})$ compared with rice $(430 \mathrm{Mb})$ [34]. Our Micro-Tom BAC coverage is reasonable, taking into account the scale of the BAC library used.

Micro-Tom has been considered as a model cultivar to promote functional genomics studies of tomato by taking advantage of its characteristics. Currently, many tools and platforms have been developed, and some of these are already available to the research community. The present study characterized the overall polymorphisms found between MicroTom BESs and the reference tomato "Heinz 1706" genome. In addition, two possible genome rearrangement events, on chromosome 2 and chromosome 3, were observed (Table 2). In the case of translocation and inversion on chromosome 3 , a gene annotated as reverse transcriptase was found in the flanking region (Solyc03g104840.1). We speculate that this region was translocated by the activity of a retrotransposon, as it was in the case of SUN. Enhanced expression of SUN caused by a gene duplication event mediated by the retrotransposon Rider led to an elongated fruit shape [35]. In the future, we plan to sequence the entire BAC and expect that this will help us to characterize these events in more detail. In the case of the other rearrangement possibility, on chromosome 2, we could not find any trace of a retrotransposon. Since these rearrangements took place in euchromatin, which is rich in genes, these regions could represent an interesting target to investigate their possible effects on phenotypic variation between Micro-Tom and the reference tomato.

We mapped the polymorphisms and depicted them, alongside maps showing covered regions and gaps, in Figure 3. On chromosomes 2, 5, and 11, polymorphisms seemed to be concentrated in the heterochromatic regions; however, this tendency was not clearly observed in the other chromosomes. For the other regions, the polymorphism discovery rate seemed to be somehow correlated with the BAC coverage. Although our analysis indicated little possibility of large-scale genome rearrangement between Micro-Tom and "Heinz 1706" (Table 2), this uneven polymorphism distribution suggests the existence of highly divergent chromosomal regions. The gaps in the hypothetical Micro-Tom BAC contigs could have resulted from low coverage of the BAC library, but the occurrence of chromosomal segments specific to either Micro-Tom or "Heinz 1706" is also possible. The ongoing Micro-Tom genome sequencing and de novo assembly of the Micro-Tom genome will clarify the genome structure in detail, enabling a more solid assessment of the differences between Micro-Tom and "Heinz 1706."

We had previously developed SNP markers among several cultivated tomatoes [12]. By selecting SNPs through in silico analysis using public EST information and previously developed SSR markers, 1,137 markers were obtained and successfully mapped on linkage groups between Micro-Tom 


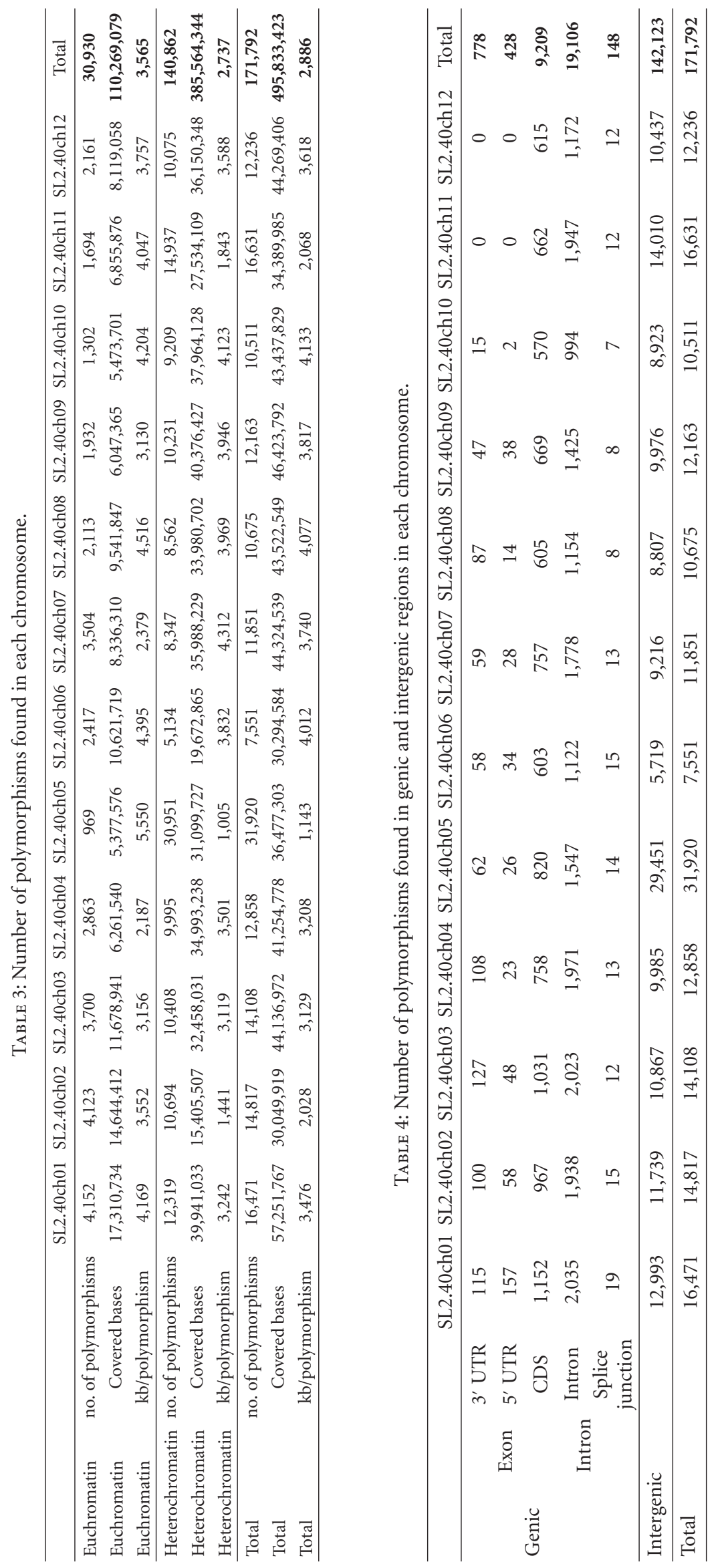




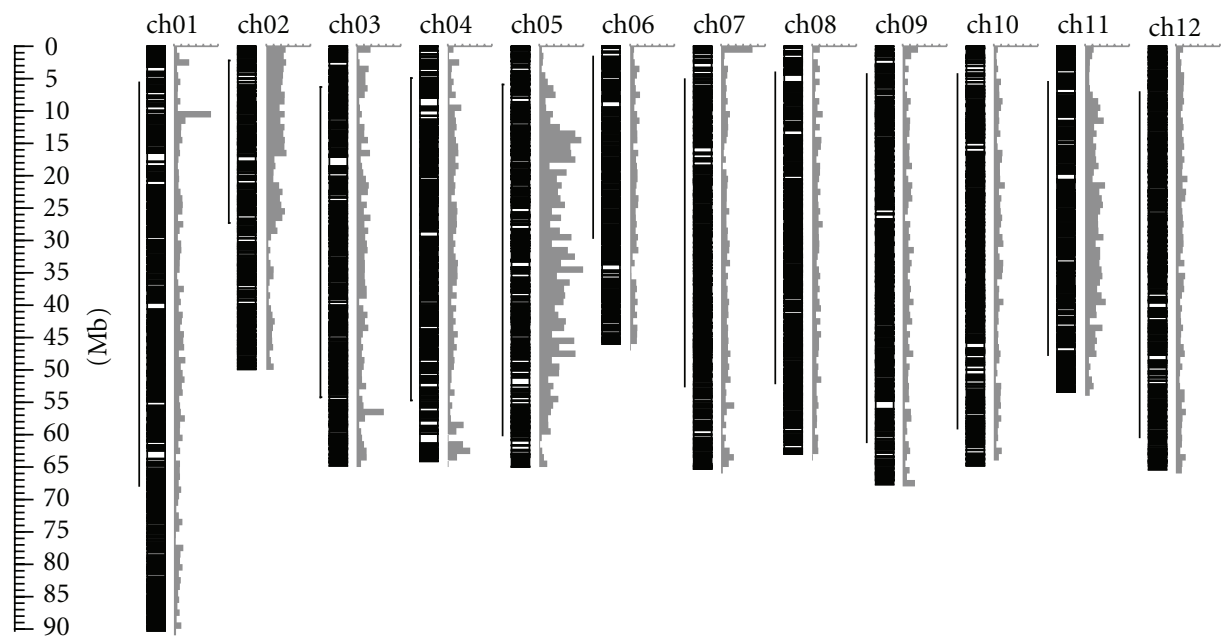

FIGURE 3: Micro-Tom BAC coverage with respect to the "Heinz 1706" chromosomes and detected polymorphisms. Black boxes indicate covered regions, and white boxes indicate gaps. Bars represent heterochromatic regions. The scale bars for polymorphisms indicate the number of SNPs or Indels per megabase (200 polymorphisms/scale).

and either Ailsa Craig or M82. In the present study, we identified 171,792 SNPs and Indels and mapped them on 12 chromosomes. The average density was 1 SNP per 3,565 bp in euchromatin and 1 SNP per 2,886 bp in the genome in general (including both euchromatin and heterochromatin). Previously, large-scale Micro-Tom full-length cDNA analysis and comparison of exon regions with those on the "Heinz 1706 " genome revealed a mean sequence mismatch of $0.061 \%(1 / 1,640 \mathrm{bp})$ [36]. One possible explanation for the difference is the quality of the reference "Heinz 1706" genome sequence used in the two studies. We used the published version of the "Heinz 1706" genome sequence, which has higher coverage, giving rise to greater accuracy, although our selection may still contain sequence errors because BESs are single-pass sequences.

The information provided in this study will be useful in the development of DNA markers between Micro-Tom and cultivated tomatoes, which will facilitate a better understanding of the physiological and metabolic differences between them. It would also be useful in the genetic mapping of Micro-Tom mutants through the generation of $\mathrm{F}_{2}$ segregating populations.

\section{Authors' Contribution}

E. Asamizu and K. Shirasawa equally contributed to this work.

\section{Acknowledgments}

The authors are grateful to Shinobu Nakayama and Akiko Watanabe for technical assistances. Construction of the Micro-Tom BAC library was supported by Japan Solanaceae Consortium (JSOL). This work was supported by the Genome Information Upgrading Program "Micro-Tom BAC-end sequencing" of the National BioResource Project in 2009.

\section{References}

[1] K. Arumuganathan and E. D. Earle, "Nuclear DNA content of some important plant species," Plant Molecular Biology Reporter, vol. 9, no. 3, pp. 208-218, 1991.

[2] Tomato Genome Consortium, "The tomato genome sequence provides insights into fleshy fruit evolution," Nature, vol. 485, no. 7400, pp. 635-641, 2012.

[3] M. R. Foolad, "Genome mapping and molecular breeding of tomato," International Journal of Plant Genomics, vol. 2007, Article ID 64358, 52 pages, 2007.

[4] I. Paran, I. Goldman, S. D. Tanksley, and D. Zamir, "Recombinant inbred lines for genetic mapping in tomato," Theoretical and Applied Genetics, vol. 90, no. 3-4, pp. 542-548, 1995.

[5] T. M. Fulton, J. C. Nelson, and S. D. Tanksley, "Introgression and DNA marker analysis of Lycopersicon peruvianum, a wild relative of the cultivated tomato, into Lycopersicon esculentum, followed through three successive backcross generations," Theoretical and Applied Genetics, vol. 95, no. 5-6, pp. 895-902, 1997.

[6] A. Frary, T. C. Nesbitt, A. Frary et al., "fw2.2: a quantitative trait locus key to the evolution of tomato fruit size," Science, vol. 289, no. 5476, pp. 85-88, 2000.

[7] J. Liu, J. Van Eck, B. Cong, and S. D. Tanksley, "A new class of regulatory genes underlying the cause of pear-shaped tomato fruit," Proceedings of the National Academy of Sciences of the United States of America, vol. 99, no. 20, pp. 13302-13306, 2002.

[8] B. Cong, L. S. Barrero, and S. D. Tanksley, "Regulatory change in YABBY-like transcription factor led to evolution of extreme fruit size during tomato domestication," Nature Genetics, vol. 40, no. 6, pp. 800-804, 2008.

[9] H. Xiao, N. Jiang, E. Schaffner, E. J. Stockinger, and E. Van Der Knaap, "A retrotransposon-mediated gene duplication underlies morphological variation of tomato fruit," Science, vol. 319, no. 5869, pp. 1527-1530, 2008.

[10] C. M. Rick, E. Kesicki, J. F. Fobes, and M. Holle, "Genetic and biosystematic studies on two new sibling species of Lycopersicon from interandean Peru," Theoretical and Applied Genetics, vol. 47, no. 2, pp. 55-68, 1976. 
[11] K. Shirasawa, E. Asamizu, H. Fukuoka et al., "An interspecific linkage map of SSR and intronic polymorphism markers in tomato," Theoretical and Applied Genetics, vol. 121, no. 4, pp. 731-739, 2010.

[12] K. Shirasawa, S. Isobe, H. Hirakawa et al., "SNP discovery and linkage map construction in cultivated tomato," DNA Research, vol. 17, no. 6, pp. 381-391, 2010.

[13] R. Meissner, Y. Jacobson, S. Melamed et al., "A new model system for tomato genetics," Plant Journal, vol. 12, no. 6, pp. 1465-1472, 1997.

[14] E. Emmanuel and A. A. Levy, "Tomato mutants as tools for functional genomics," Current Opinion in Plant Biology, vol. 5, no. 2, pp. 112-117, 2002.

[15] H. J. Sun, S. Uchii, S. Watanabe, and H. Ezura, "A highly efficient transformation protocol for Micro-Tom, a model cultivar for tomato functional genomics," Plant and Cell Physiology, vol. 47, no. 3, pp. 426-431, 2006.

[16] G. J. Bishop, K. Harrison, and J. D. G. Jones, "The tomato Dwarf gene isolated by heterologous transposon tagging encodes the first member of a new cytochrome P450 family," Plant Cell, vol. 8, no. 6, pp. 959-969, 1996.

[17] E. Martí, C. Gisbert, G. J. Bishop, M. S. Dixon, and J. L. García-Martínez, "Genetic and physiological characterization of tomato cv. Micro-Tom," Journal of Experimental Botany, vol. 57, no. 9, pp. 2037-2047, 2006.

[18] S. Watanabe, T. Mizoguchi, K. Aoki et al., "Ethylmethanesulfonate (EMS) mutagenesis of Solanum lycopersicum cv. MicroTom for large-scale mutant screens," Plant Biotechnology, vol. 24, no. 1, pp. 33-38, 2007.

[19] C. Matsukura, I. Yamaguchi, M. Inamura et al., "Generation of gamma irradiation-induced mutant lines of the miniature tomato (Solanum lycopersicum L.) cultivar 'Micro-Tom,'” Plant Biotechnology, vol. 24, no. 1, pp. 39-44, 2007.

[20] T. Saito, T. Ariizumi, Y. Okabe et al., "TOMATOMA: a novel tomato mutant database distributing micro-tom mutant collections," Plant and Cell Physiology, vol. 52, no. 2, pp. 283296, 2011.

[21] L. A. Mueller, S. D. Tanskley, J. J. Giovannoni et al., "The tomato sequencing project, the first cornerstone of the International Solanaceae Project (SOL)," Comparative and Functional Genomics, vol. 6, no. 3, pp. 153-158, 2005.

[22] S. Katagiri, J. Wu, Y. Ito et al., "End sequencing and chromosomal in silico mapping of BAC clones derived from an indica rice cultivar, Kasalath," Breeding Science, vol. 54, no. 3, pp. 273-279, 2004.

[23] T. R. Silva Figueira, V. Okura, F. R. da Silva et al., "A BAC library of the SP80-3280 sugarcane variety (saccharum sp.) and its inferred microsynteny with the sorghum genome," BMC Research Notes, vol. 5, p. 185, 2012.

[24] S. K. Sehgal, W. Li, P. D. Rabinowicz et al., "Chromosome arm-specific BAC end sequences permit comparative analysis of homoeologous chromosomes and genomes of polyploid wheat," BMC Plant Biology, vol. 12, p. 64, 2012.

[25] M. K. Sharma, R. Sharma, P. Cao et al., "A genome-wide survey of switchgrass genome structure and organization," PLoS One, vol. 7, no. 4, Article ID e33892, 2012.

[26] H. Wang, R. V. Penmetsa, M. Yuan et al., "Development and characterization of BAC-end sequence derived SSRs, and their incorporation into a new higher density genetic map for cultivated peanut (Arachis hypogaea L.)," BMC Plant Biology, vol. 12, p. 10, 2012.

[27] B. Ewing, L. Hillier, M. C. Wendl, and P. Green, "Base-calling of automated sequencer traces using phred. I. Accuracy assessment," Genome Research, vol. 8, no. 3, pp. 175-185, 1998.
[28] B. Ewing and P. Green, "Base-calling of automated sequencer traces using phred. II. Error probabilities," Genome Research, vol. 8, no. 3, pp. 186-194, 1998.

[29] S. F. Altschul, W. Gish, W. Miller, E. W. Myers, and D. J. Lipman, "Basic local alignment search tool," Journal of Molecular Biology, vol. 215, no. 3, pp. 403-410, 1990.

[30] S. F. Altschul, T. L. Madden, A. A. Schäffer et al., "Gapped BLAST and PSI-BLAST: a new generation of protein database search programs," Nucleic Acids Research, vol. 25, no. 17, pp. 3389-3402, 1997.

[31] X. B. Zhong, P. F. Fransz, J. W. V. Eden et al., "FISH studies reveal the molecular and chromosomal organization of individual telomere domains in tomato," Plant Journal, vol. 13, no. 4, pp. 507-517, 1998.

[32] Y. Wang, X. Tang, Z. Cheng, L. Mueller, J. Giovannoni, and S. D. Tanksley, "Euchromatin and pericentromeric heterochromatin: comparative composition in the tomato genome," Genetics, vol. 172, no. 4, pp. 2529-2540, 2006.

[33] J. W. Scott and B. K. Harbaugh, MICRO-TOM: A Miniature Dwarf Tomato (Circular), Agricultural Experiment Station, Institute of Food and Agricultural Sciences, University of Florida, 1989.

[34] J. Yu, S. Hu, J. Wang et al., "A draft sequence of the rice genome (Oryza sativa L. ssp. indica)," Science, vol. 296, no. 5565, pp. 79-92, 2002.

[35] H. Xiao, N. Jiang, E. Schaffner, E. J. Stockinger, and E. Van Der Knaap, "A retrotransposon-mediated gene duplication underlies morphological variation of tomato fruit," Science, vol. 319, no. 5869, pp. 1527-1530, 2008.

[36] K. Aoki, K. Yano, A. Suzuki et al., "Large-scale analysis of full-length cDNAs from the tomato (Solanum lycopersicum) cultivar Micro-Tom, a reference system for the Solanaceae genomics," BMC Genomics, vol. 11, no. 1, article no. 210, 2010. 

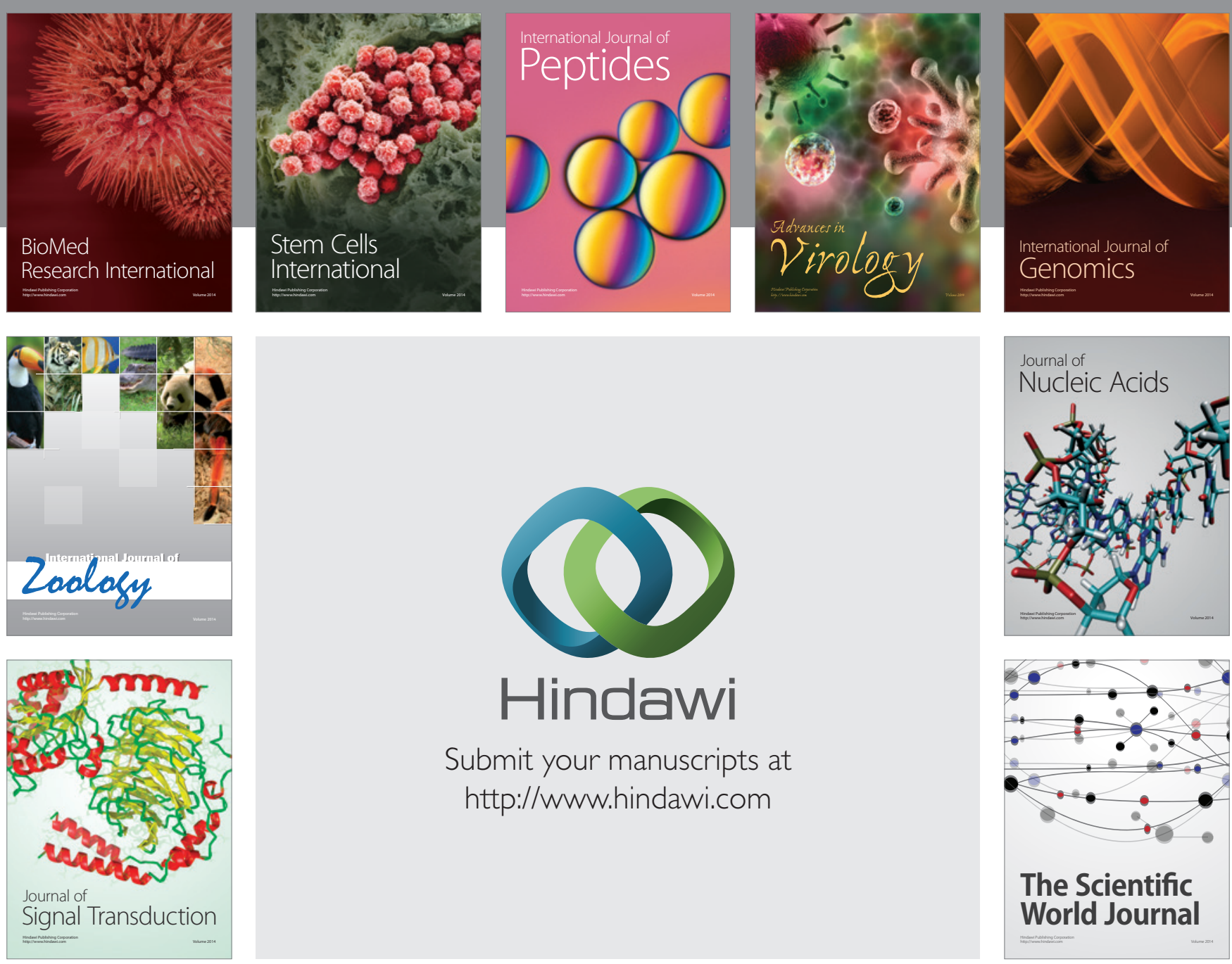

Submit your manuscripts at

http://www.hindawi.com
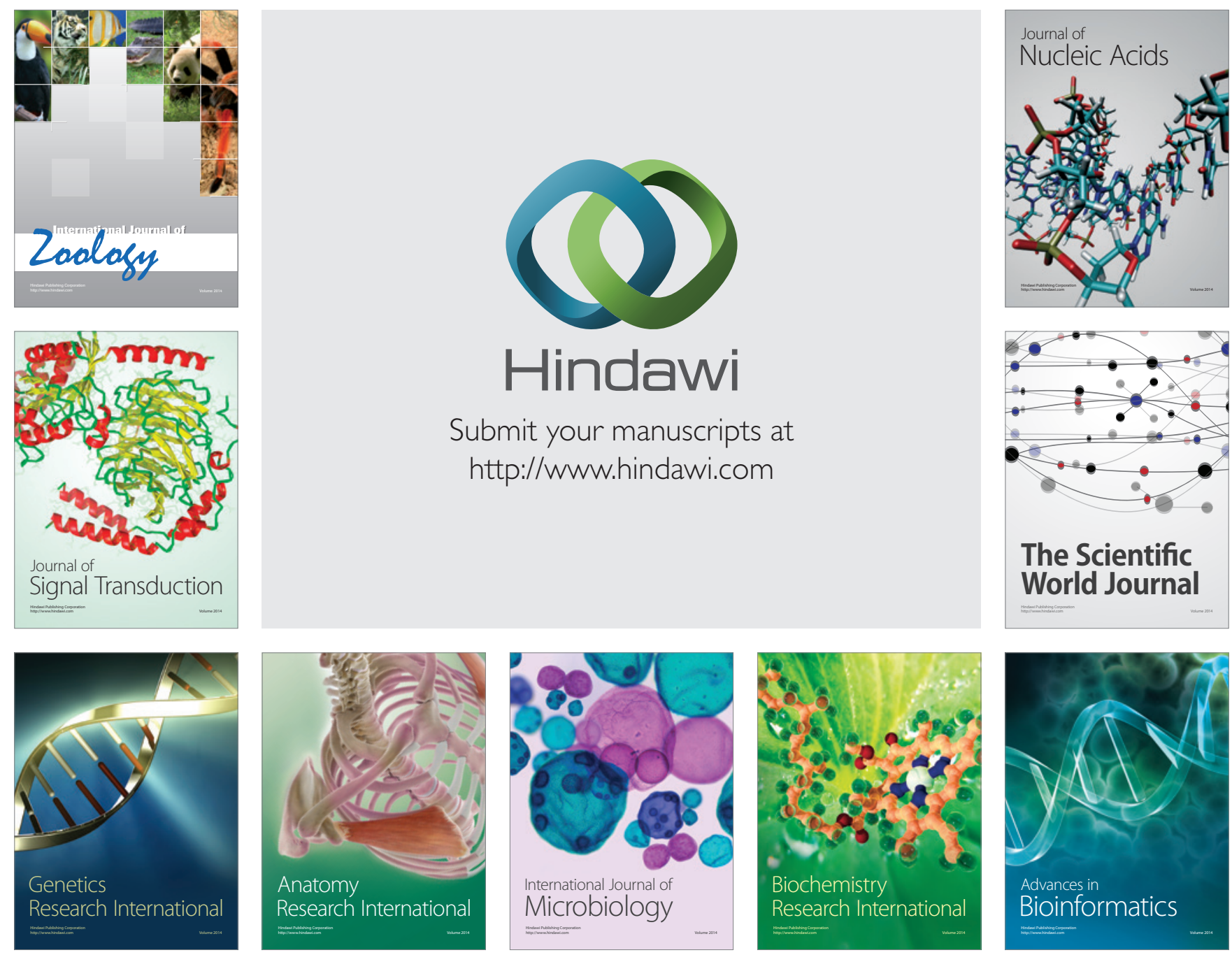

The Scientific World Journal
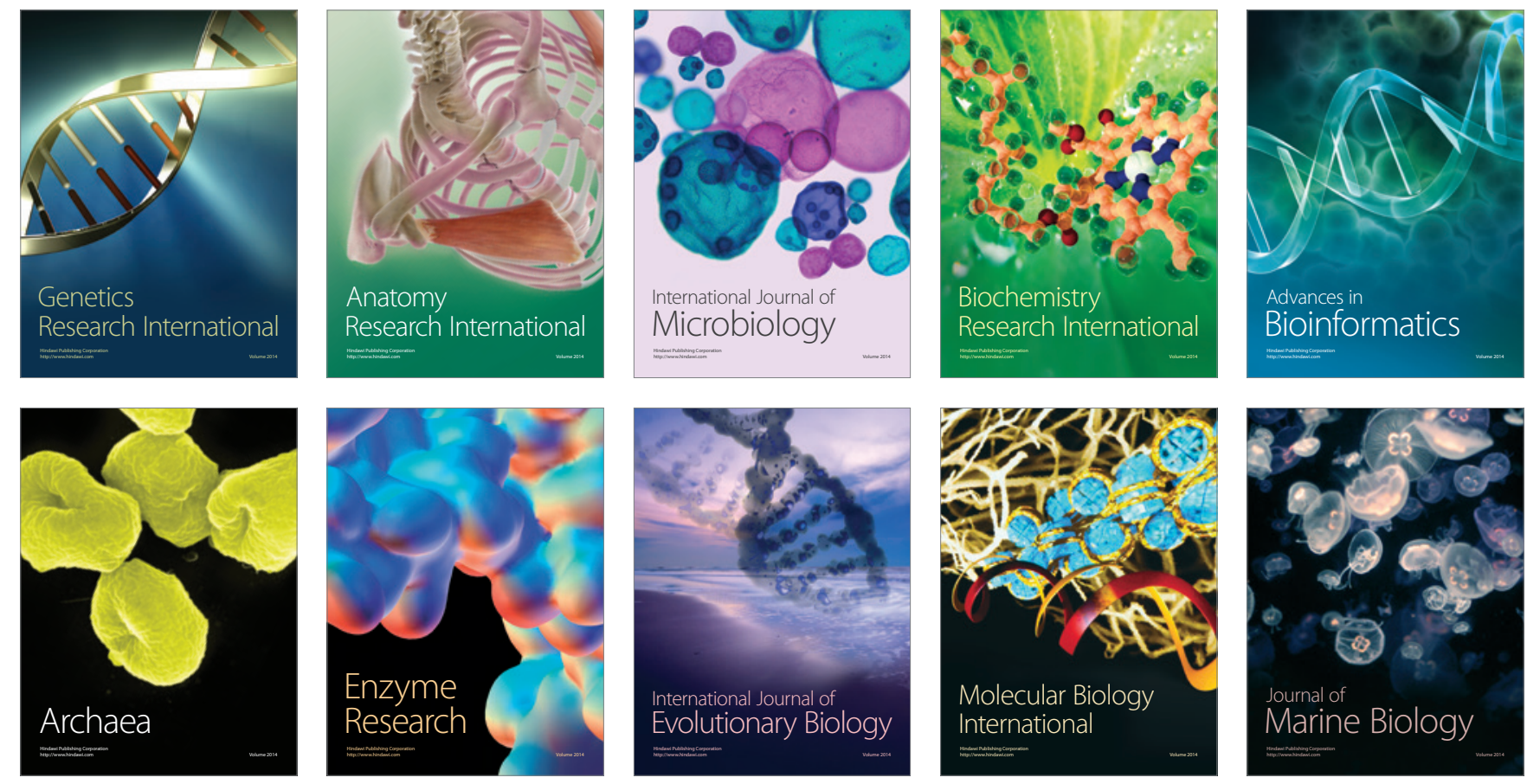